\title{
Psychopathological Comorbidities and Clinical Variables in Patients With Medication Overuse Headache
}

\author{
Simone Migliore ${ }^{1 *}$, Matteo Paolucci ${ }^{2}$, Livia Quintiliani ${ }^{3}$, Claudia Altamura ${ }^{2}$, Sabrina Maffi ${ }^{1}$, \\ Giulia D'Aurizio ${ }^{4}$, Giuseppe Curcio ${ }^{4}$ and Fabrizio Vernieri ${ }^{2}$ \\ ${ }^{1}$ Huntington and Rare Diseases Unit, Fondazione IRCCS Casa Sollievo della Sofferenza, San Giovanni Rotondo, Italy, ${ }^{2}$ UOS \\ Cefalee e Neurosonologia, Neurology, University Campus Bio-Medico, Rome, Italy, ${ }^{3}$ Psychology Service, University Campus \\ Bio-Medico, Rome, Italy, ${ }^{4}$ Department of Biotechnological and Applied Clinical Sciences, University of L'Aquila, L'Aquila, Italy
}

\section{OPEN ACCESS}

Edited by:

Marina De Tommaso, University of Bari Aldo Moro, Italy

Reviewed by: Giorgio Sandrini,

University of Pavia, Italy

Luigi Alberto Pini,

University of Modena and Reggio

Emilia, Italy

*Correspondence:

Simone Migliore

sim.migliore@gmail.com

Specialty section:

This article was submitted to

Health,

a section of the journal

Frontiers in Human Neuroscience

Received: 09 June 2020 Accepted: 06 November 2020 Published: 27 November 2020

Citation:

Migliore S, Paolucci M, Quintiliani L, Altamura C, Maffi S, D'Aurizio G, Curcio $G$ and Vernieri $F$

(2020) Psychopathological Comorbidities and Clinical Variables in Patients With Medication Overuse

Headache.

Front. Hum. Neurosci. 14:571035. doi: 10.3389/fnhum.2020.571035
The psychopathological profile of patients with medication overuse headache $(\mathrm{MOH})$ appears to be particularly complex. To better define it, we evaluated their performance on a targeted psychological profile assessment. We designed a case-control study comparing $\mathrm{MOH}$ patients and matched healthy controls $(\mathrm{HC})$. Headache frequency, drug consumption, HIT-6, and MIDAS scores were recorded. All participants filled in the following questionnaires: Beck Depression Inventory-II Edition (BDI-2), trait subtest of State-Trait Anxiety Inventory (STAI-Y), Difficulties in Emotion Regulation Scale (DERS), Barratt Impulsiveness Scale (BIS-11), Toronto Alexithymia Scale (TAS-20). The primary endpoint was to establish if $\mathrm{MOH}$ patients have an altered psychopathological profile. The secondary endpoint was to establish whether the worst profile correlates with the worsening of headache and disability measures. We enrolled 48 consecutive $\mathrm{MOH}$ patients and $48 \mathrm{HC} . \mathrm{MOH}$ patients showed greater difficulty in recognition/regulation of emotions (DERS, TAS-20), depression (BDI-2), anxiety (STAI-Y), and impulsiveness (BIS-11). We found a positive correlation among DERS, BDI-2, STAI-Y, and BIS scores and MIDAS and HIT-6 scores and among DERS and headache frequency and drug consumption. $\mathrm{MOH}$ patients showed a high rate of emotion regulation difficulties, depression, and anxiety, which may negatively affect their headaches. The ability to regulate/recognize emotions may play a central role in sustaining medication overuse.

Keywords: emotion regulation, emotion recognition (ER), psychopatological profile, medication overuse headache (MOH), behavioral approach

\section{INTRODUCTION}

The daily or almost daily frequent use of symptomatic drugs in patients with high frequency or chronic migraine, and less frequently with chronic tension-type headache, leads to the development of medication overuse headache (MOH).

The psychopathological profile of patients with $\mathrm{MOH}$ is very complex: together with mood and anxiety disorders, it can be observed as tending to obsessive-compulsive disorders and the occurrence of dependance-related behavior (Cupini et al., 2009; Radat and LanteriMinet, 2010; Lampl et al., 2016), and it has yet been suggested that a psychological profile assessment should be included in patients' evaluation (Sarchielli et al., 2016). 
A negative prognostic value for psychiatric comorbidities has been suggested putting forward the hypothesis that these can represent a risk factor for the evolution of episodic into chronic headaches (Radat and Swendsen, 2005; Guidetti et al., 2010). Psychopathological disturbances are also seen as a potential predictor of relapse and poor response to treatment, and this can, in turn, complicate headache management facilitating $\mathrm{MOH}$ development (Cupini et al., 2009; Radat and LanteriMinet, 2010). Finally, some studies raised hypotheses about the potential comorbidity between psychiatric disorders and chronic headaches, but the presence of psychiatric disorders in $\mathrm{MOH}$ patients has been verified only in some of these (Buse et al., 2013; Sarchielli et al., 2016).

Our study aimed at evaluating the prevalence of psychopathological profiles in $\mathrm{MOH}$ patients through a comprehensive psychopathological battery to assess depressive symptoms and anxiety disorders, emotions' recognition and elaboration, and impulsiveness' level. We also investigated potential correlations between the psychopathological profile and some clinical variables (i.e., headache frequency, drug consumption, the impact of headaches on abilities of daily living). We expected $\mathrm{MOH}$ patients to show higher scores in psychopathological questionnaires compared with healthy controls (HC). Moreover, we hypothesized that psychopathological scores correlate with different clinical variables (i.e., monthly days of headache, medications taken per month, disease duration, and migraine-related functional disability).

\section{MATERIALS AND METHODS}

\section{Participants}

We designed a case-control study comparing patients affected by $\mathrm{MOH}$ with $\mathrm{HC}$, regarding possible differences in psychopathological profile. During the enrollment period (November 2015-May 2017), participation in the study was proposed to every outpatient with a $\mathrm{MOH}$ diagnosis that visited our Headache Center. $\mathrm{MOH}$ was diagnosed according to the International Classification of Headache Disorders, 3rd edition, beta version [Headache Classification Committee of the International Headache Society (IHS), 2013], based on clinical characteristics and the headache frequency resulting from personal headache daily diaries. Every other headache diagnosis was based on the ICHD criteria. Patients with suspected symptomatic headaches were investigated and excluded if needed.

Inclusion criteria for patients were age $\geq 18$ years old, and fulfilling the ICHD 3rd edition, beta version criteria for $\mathrm{MOH}$. Exclusion criteria were secondary headaches, and lack of inclusion criteria.

HC matched by age and gender were recruited among employees of the University Campus Bio-Medico. They all were free of medications at the moment of the assessment. Moreover, based on a clinical interview, we excluded those subjects who reported any known medical condition and neurological or psychiatric disease.

All patients had been under the care of our Headache Center for at least 3 months before the enrolment in the study and regularly completed their headache daily diary. We prescribed preventive therapy if patients were not taking it or a new one if they were. Patients were suggested not to overuse painkillers. Acetaminophen/paracetamol was allowed to treat the attacks. However, when patients were unable to refrain to take their usual symptomatic drugs, they were recommended to record in their diaries the number of triptans or NSAIDs or other analgesics they were forced to take. We reassessed patients after 3 months from the first visit. Mean headache frequency and symptomatic drug consumption in the previous 3 months were extracted from the diaries. If patients still overuse painkillers and the $\mathrm{MOH}$ diagnosis was confirmed, they had to start a bridge therapy protocol (Paolucci et al., 2017) for helping the withdrawal of symptomatic drugs. The protocol consisted of a 5-day iv infusion of saline solution $\mathrm{NaCl} \quad 0.9 \% 250 \mathrm{ml}$ with methylprednisolone $125 \mathrm{mg}$ plus diazepam $10 \mathrm{mg}$, infused at $100 \mathrm{ml} / \mathrm{h}$, and daily monitoring in a Day Hospital setting. Patients had not to take the overused symptomatic drug(s) and at the end of these 5 days received a new prophylactic therapy.

After informed consent was given, patients and controls were enrolled. At the moment of inclusion in the study, patients were asked to fill in a set of questionnaires to assess psychological profile, as described afterward. We also asked the patients to fill in Headache Impact Test (HIT-6; Bayliss et al., 2003) and Migraine Disability Assessment (MIDAS; Stewart et al., 2001) scores.

The primary endpoint was to establish if $\mathrm{MOH}$ patients have an altered psychopathological profile as compared to HC.

The secondary endpoint was to establish whether a worst psychopathological profile correlates with the worsening of headache impact and disability measures in $\mathrm{MOH}$ patients.

This study was designed following the ethical principles of the Declaration of Helsinki and all participants were asked to sign an informed consent. The study was approved by Campus Bio-Medico University Ethics Committee, approval number 4418, and registered at AIFA (Italian Drug Agency) with number Eudract 2017-004606-18.

\section{Psychopathological Assessment}

Patients, before starting bridge therapy protocol, and $\mathrm{HC}$ filled in a set of questionnaires to assess psychological profile, composed by:

1. Beck Depression Inventory-II Edition (BDI-2; Beck et al., 1996), a 21 multiple-choice questions self-report inventory to measure the severity of depression.

2. Trait subtest of State-Trait Anxiety Inventory (STAI-Y; Spielberger et al., 1983), a 20 multiple-choice items self-report questionnaire for measuring anxiety disorder.

3. Difficulties in Emotion Regulation Scale (DERS; Sighinolfi et al., 2010), a 36 items self-report questionnaire designed to measure multiple aspects of emotion dysregulation. 
The scale provides both a total score and scores on six subscales: non-acceptance of emotional responses (NONACCEPTANCE), difficulties engaging in goal-directed behavior (GOALS), impulse control difficulties (IMPULSE), lack of emotional awareness (AWARENESS), limited access to emotion regulation strategies (STRATEGIES), and lack of emotional clarity (CLARITY).

4. Barratt Impulsiveness Scale (BIS-11; Fossati et al., 2001) a 30 multiple-choice items self-report questionnaire for measure impulsiveness. The questionnaire provides a total score and $3 \mathrm{~s}$-order factors, attention, motor, and non-planning impulsiveness.

5. Toronto Alexithymia Scale (TAS-20; Bressi et al., 1996) a 20 multiple-choice items self-report inventory for evaluating difficulties to identify and describe emotions. The scale provides a total score and three subscores, related to Difficulty Identifying Feelings (DIF), Difficulty Describing Feelings (DDF), and Externally and Oriented Thinking (EOT).

\section{Statistical Analysis}

To better describe the psychopathological profile of patients and to highlight possible emotional dysregulation we decided to run a post hoc analysis partially based on previous data (Migliore et al., 2018). Chi-square test and Student's $t$-test were run to assess the statistical difference between $\mathrm{MOH}$ patients and $\mathrm{HC}$ for sex and age distribution. Baseline headache measures were expressed as mean (SD) or median (IQR) depending on the variable distribution.

For the primary endpoint, to examine differences in experimental groups test performances, all dependent variables, obtained from psychopathological assessment scores (BDI-2, DERS, STAI-Y, BIS-11, TAS-20), were submitted to one-way ANOVA directly comparing the scores of two different groups ( $\mathrm{MOH}$ vs. HC).

For the secondary endpoint, to highlight possible relationships between clinical variables (headache impact and disability measures: headache frequency, drug consumption HIT-6, MIDAS total score) and $\mathrm{MOH}$ psychopathological questionnaire performance, we initially performed bivariate correlation analysis. Taken into account that data did not respect the assumption of linearity and normality, we used Spearman's correlation coefficient.

Alpha level was fixed to $\leq 0.05$. All statistical analyses were performed using SPSS 25.

\section{RESULTS}

From November 2015 to May 2017 we enrolled 48 consecutive patients with $\mathrm{MOH}$ diagnosis (see Table 1 for clinical and demographic characteristics). We then enrolled 48 matched HC (Table 1).

Patients showed a mean of 24.1 days $( \pm 6.4)$ of headache per month and a median of 40 symptomatic medications taken per month (IQR: 36; minimum 12 and maximum 315). The mean HIT-6 score was $67.4( \pm 5.6)$, the mean of MIDAS total score was $88.8( \pm 71.3)$, MIDAS-A score was $61.2( \pm 28.3)$ and MIDAS-B score was $8.2( \pm 1.3)$. We found no significant difference between men and women.

The group comparison showed that $\mathrm{MOH}$ patients and HC groups differed significantly in terms of total score of emotion regulation difficulties (DERS total score; $F_{(1,94)}=17.68$; $\left.p<0.00001 ; \eta_{\mathrm{p}}^{2}=0.15\right)$, depression $\left(\mathrm{BDI}-2 ; F_{(1,94)}=19.04 ;\right.$ $p<0.0001 ; \eta_{\mathrm{p}}^{2}=0.16$ ), alexithymia (TAS-20 total score; $\left.F_{(1,94)}=9.05 ; p=0.003 ; \eta_{\mathrm{p}}^{2}=0.08\right)$, anxiety (STAI-Y; $\left.F_{(1,94)}=23.18 ; p<0.00001 ; \eta_{\mathrm{p}}^{2}=0.19\right)$. No difference was highlighted between groups in term of impulsiveness (BIS11 total score).

When comparing the subscales of DERS, significant differences were observed in the Nonaccept score $\left(F_{(1,94)}=6.93\right.$; $\left.p=0.01 ; \eta_{\mathrm{p}}^{2}=0.06\right)$, Impulse score $\left(F_{(1,94)}=6.96 ; p=0.01\right.$; $\left.\eta_{\mathrm{p}}^{2}=0.06\right)$, Aware score $\left(F_{(1,94)}=6.55 ; p=0.01 ; \eta_{\mathrm{p}}^{2}=0.06\right)$, Strategies score $\left(F_{(1,94)}=16.28 ; p<0.00001 ; \eta_{\mathrm{p}}^{2}=0.14\right)$, and Clarity score $\left(F_{(1,94)}=7.31 ; p=0.008 ; \eta_{\mathrm{p}}^{2}=0.07\right)$; no differences emerged in Goal score. Regarding to BIS subscales, significant differences were observed in the Attention score $\left(F_{(1,94)}=7.7\right.$; $\left.p=0.006 ; \eta_{\mathrm{p}}^{2}=0.07\right)$; no differences highlighted in motor and no planning. Regarding to TAS-20 subscales, we observed statistical difference in DIF subscales $\left(F_{(1,94)}=16.47 ; p<0.00001\right.$; $\left.\eta_{\mathrm{p}}^{2}=0.15\right)$; no differences emerged in DDF and EOT subscores. The full details of the comparison results are shown in Table 2.

We found a significant correlation between basal HIT-6 score and depression (BDI-2; rs $=0.58 ; p<0.0001$ ), impulsivity both Attention (BIS-11; rs $=0.43 ; p \leq 0.002$ ) and total BIS-11 scores ( $\mathrm{rs}=0.33 ; p=0.02$ ), regulation of emotions (DERS nonaccept; $\mathrm{rs}=0.4 ; p=0.006$; DERS goals; $\mathrm{rs}=0.6 ; p<0.0001$; DERS strategies; $\mathrm{rs}=0.53 ; p<0.0001$; DERS clarity $\mathrm{rs}=0.45$; $p=0.001$; DERS total; rs $=0.58 ; p<0.0001)$, trait anxiety (trait subtest of STAI-Y; $\mathrm{rs}=0.6 ; p<0.0001$ ), and, finally, alexithymia (TAS-20 DIF; rs $=0.4 ; p=0.006$ ). Moreover, we found a significant correlation between headache frequency and regulation of emotions (DERS Aware; $r s=0.3 ; p=0.04$ ) and between number of medications and regulation of emotions (DERS Aware; rs $=0.3 ; p=0.03$ ). Finally, our analysis showed a significant correlation between basal total MIDAS Total score and depression (BDI-2; rs $=0.3 ; p=0.04$ ) and between MIDAS-B and trait anxiety (trait subtest of STAI-Y; $r s=0.28 ; p=0.04$ ). No correlation was found between psychopathological scores and disease duration.

\section{DISCUSSION}

Our study showed a significant difference in many psychopathological scales scores between $\mathrm{MOH}$ patients and HC subjects. Particularly, we demonstrated a high rate of depression, anxiety, and impulsiveness associated with a specific difficulty in recognizing and regulating emotions. Moreover, we found a positive correlation among psychopathological scales scores and both MIDAS and HIT- 6 questionnaires, assessing the degree of migraine-related functional disability, showing that psychological comorbidities together with $\mathrm{MOH}$ negatively affect patients' activities of daily living. Finally, we found a positive correlation between the DERS Aware subscore and some clinical 
TABLE 1 | Demographic and clinical characteristics of the study sample.

\begin{tabular}{|c|c|c|c|}
\hline & MOH patients $(n=48)$ & Healthy controls $(n=48)$ & $p$ \\
\hline Sex & $\mathrm{F}: 38-\mathrm{M}: 10$ & $\mathrm{~F}: 37-\mathrm{M}: 11$ & $p=0.805$ \\
\hline Age in years (mean $\pm S D$ ) & $47.7 \pm 12.1$ & $46.8 \pm 10.71$ & $p=0.702$ \\
\hline Disease duration in years (mean $\pm \mathrm{SD}$ ) & $26.1 \pm 15.1$ & - & \\
\hline Monthly days of headache (mean \pm SD) & $24.1 \pm 6.4$ & - & \\
\hline Monthly drugs intake Median (min-max) & $40(12-315)$ & - & \\
\hline MIDAS-total (mean \pm SD) & $88.8 \pm 71.3$ & & \\
\hline MIDAS-A (mean \pm SD) & $61.2 \pm 28.3$ & - & \\
\hline MIDAS-B (mean \pm SD) & $8.2 \pm 1.3$ & & \\
\hline HIT-6 (mean \pm SD) & $67.4 \pm 5.6$ & - & \\
\hline
\end{tabular}

Statistical comparisons refer to Chi-Square for the sex composition of the samples and Student's t-test for mean age.

TABLE 2 | Participants' scores across the outcome variables.

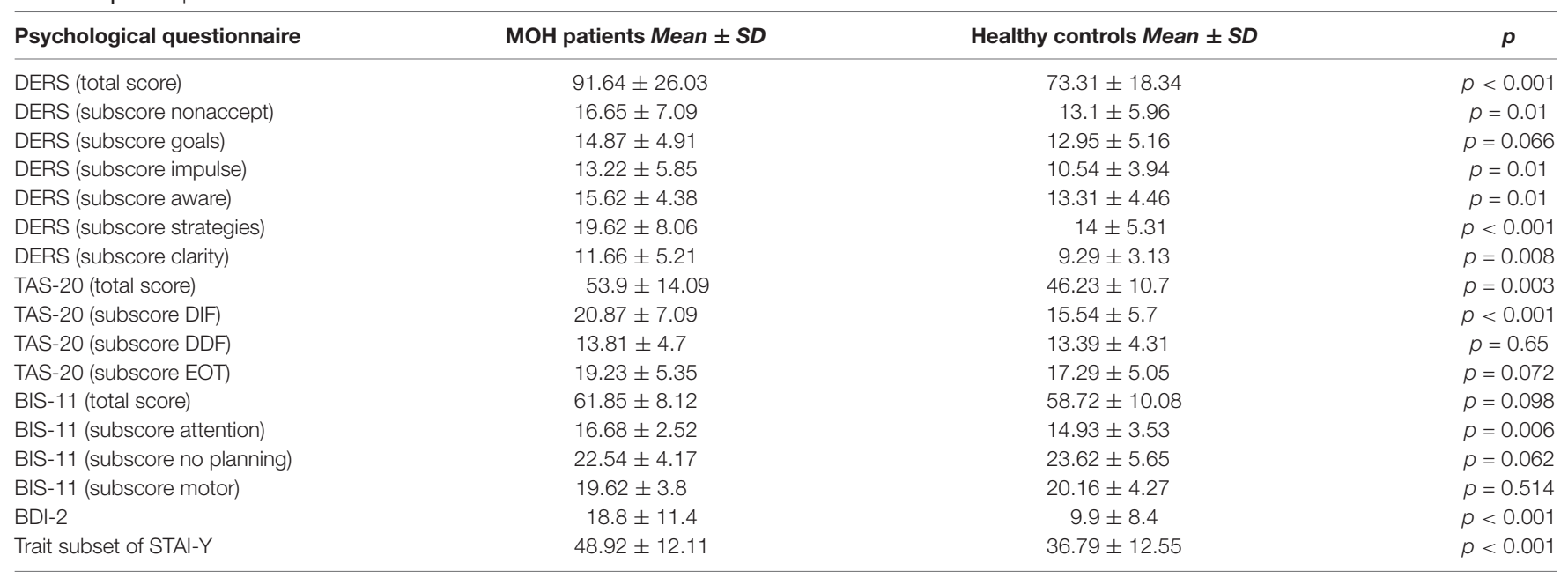

Note: $\mathrm{MOH}$, Medication Overuse Headache; DERS, Difficulties in Emotion Regulation Scale; NONACCEPT, non-acceptance of emotional responses; GOALS, difficulties engaging in goal-directed behavior; IMPULSE, impulse control difficulties; AWARE, lack of emotional awareness; STRATEGIES, limited access to emotion regulation strategies; CLARITY, lack of emotional clarity; TAS-20, Toronto Alexitimia Scale-20 item; DIF, Difficulty Identifying Feeling; DDF, difficulty describing feelings; EOT, externally oriented thinking; BIS, Barratt Impulsiveness Scale; BDI-2, Beck Depression Inventory 2; STAI-Y, State-Trait Anxiety Inventory.

variables, specifically headache frequency and the number of painkillers, but not with disease duration. This observation suggests that some of the psychological aspects evaluated are constitutional in patients with $\mathrm{MOH}$ and not the consequence of a long-standing pain condition. This relation between emotional dysregulation and pain intensity/analgesic consumption shows that the impairment in recognition/regulation of emotions producing an important dysfunctional behavior hugely impacts on disability from headache, regardless of the disease chronicity. To our knowledge, the present study is the first aiming to explore the emotions' regulation abilities in a population of $\mathrm{MOH}$ patients. Besides, this is the first attempt also to explore the relationship between emotion regulation abilities and depression and anxiety in a $\mathrm{MOH}$ patients' sample. Emotion regulation is the process of managing one's emotions, but at the same time regards the "when" and the "how" individuals experience or express the emotions (Ciarrochi et al., 2001). Such a process involves both negative and positive emotions and when it works successfully can guarantee good mental health, as recently shown (Eftekhari et al., 2009). Difficulties in recognizing and regulating emotions have emerged in other neurological diseases, i.e Huntington Disease (Zarotti et al., 2018) and Multiple Sclerosis (Migliore et al., 2019). In the last years, also great attention has been paid to the nighttime involvement of emotional experience during dreaming, that correlated with volumetric and ultrastructural brain measures (e.g., De Gennaro et al., 2011). These findings suggested that difficulties in emotional skills (recognizing and regulating) may represent a precursor of more general cognitive impairment that could negatively impact daily life activities. Different reviews and meta-analysis (Di Tella and Castelli, 2016; Koechlin et al., 2018; Aaron et al., 2019) highlight as a recent growing body of researches is interested to evaluate emotion regulation's role in different chronic pain (i.e., Complex Regional Pain Syndrome and Low Back Pain, Temporomandibular Disorders, Fibromyalgia, et cetera). These studies show significant emotion regulation difficulties in different types of chronic pain conditions. Emotion dysregulation may be an important risk factor in the development and maintenance of chronic pain and it is associated with many clinical (i.e., pain intensity) and psychological variables (anxiety and depression).

In $\mathrm{MOH}$ patients, it is possible to hypothesize that the chronic, almost daily, headache produces negative emotions. The $\mathrm{MOH}$ patient has difficulty coping with negative emotions (impairment 

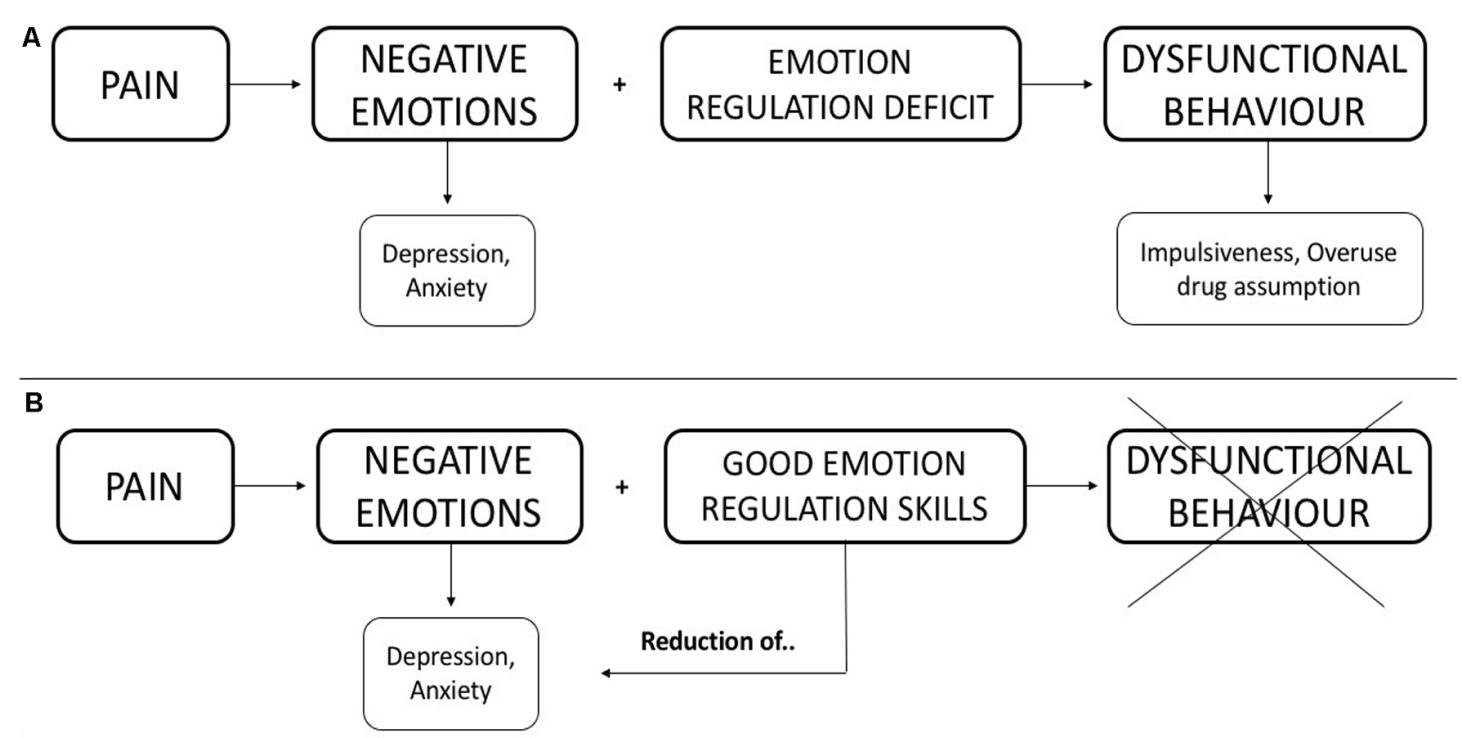

FIGURE 1 | Hypothesized interaction among the discussed constructs. (A) We hypothesize that negative emotions associate with deficits in emotional regulation produce dysfunctional behavior. (B) We illustrate how good emotion regulation skills can limit dysfunctional behavior and, consequently, reduce negative emotions.

in emotion regulation abilities) and this psychological feature can represent a specific condition that may generate dysfunctional behaviors (psychopathological symptoms). Figure 1A shows a schematization of the hypothesized interaction.

Furthermore, our study confirms the high rate of depression and anxiety symptoms in $\mathrm{MOH}$ patients, as highlighted by previous researches (Lampl et al., 2016; Sarchielli et al., 2016).

Several mechanisms have been proposed to explain the comorbidity of headache and psychopathological symptoms: (a) unidirectional or bidirectional causal models; (b) shared genetic factors; and (c) environmental risk factors. Overall, the lack of clear predictive relationships between psychopathological symptoms and headache raises the possibility either of a symmetrical causal link (i.e., each disorder would be a risk factor of the other), or of a common genetic or environmental risk factor (Radat and Swendsen, 2005). Indeed, the interactive effect between environmental risk factors and genetic factors could reasonably induce the development of both the considered diseases (Radat and Swendsen, 2005). On the contrary, the relationship between depression and migraine would appear to be bidirectional, i.e., each condition would increase the incidence of the other (Breslau et al., 2003).

We hypothesize that the altered ability, both to recognize and regulate emotions may play a central role in the behavior of patients with $\mathrm{MOH}$. These altered behavioral abilities can contribute to the chronicization of head pain and to overuse of symptomatic drugs (as illustrated in Figure 1A), which is hardly treated only with a pharmacological approach.

The capability of recognizing emotions can allay a lot of negative emotions (moods) originating from headaches. In light of this, the evaluation of the psychopathological profile should be included in the general assessment of $\mathrm{MOH}$ patients. In this way, clinicians could plan an integrated treatment (both behavioral and pharmacological) to significantly improve $\mathrm{MOH}$ handling. Focusing early on the impairment of regulating emotions could have also a positive effect on anxiety-depressive symptoms and reduce dysfunctional behaviors (i.e., impulsiveness, overuse of drug assumption). We illustrated this hypothesis in Figure 1B.

Treating $\mathrm{MOH}$ patients, trying to reverse their compelling necessity to consume drugs, is a hard challenge for the headache specialist. Detoxification from $\mathrm{MOH}$ is a shared but not worldwide standardized practice used by headache units. Nevertheless, wash-out seems a useful protocol for treating medication overuse but only in the short term (Paolucci et al., 2017). There is a need for treating $\mathrm{MOH}$ comprehensively, including both a standardized pharmacological protocol and a psychological adequate approach. The battery of scales and questionnaires we used was useful in selecting patients with the highest degree of disability who might benefit from additional treatment approaches designed based on their individual profile of psychopathology.

The reason why some patients overuse acute treatments presenting $\mathrm{MOH}$ while others do not is not clearly understood. $\mathrm{MOH}$ might be related to some psychological states, such as fear and anticipatory anxiety of attacks, also defined as cephalalgophobia, The experience of recurrent severe pain may produce anticipatory anxiety for the forthcoming headaches and their consequence in terms of loss of daily activities which can be as dreadful as pain (Black et al., 2015). An alternative explanation relies on behavioral disorders, i.e., reward mechanism or compulsive disorder (Cupini et al., 2009). A drug-seeking behavior and the subsequent compulsive use of medications strongly complicate the drug withdrawal, which is the first step for treating $\mathrm{MOH}$. So far, a direct link between 
compulsive behavior and medication overuse has not been established. Understanding the underlying mechanisms of those behaviors might improve the management of $\mathrm{MOH}$.

The main limitation of our work is the lack of comparison with headaches or other pain conditions other than $\mathrm{MOH}$. Future research is needed to consider patients with other headaches (i.e., migraine headache, tension headache, or cluster headache) and without $\mathrm{MOH}$ for evaluating the potential difference in the psychopathological profile and assess whether emotional dysregulation can be transversal to different forms of headache or specific to $\mathrm{MOH}$. Moreover, since the sample size in the present analysis is rather small, prospective confirmation is needed in larger cohorts. Finally, it is necessary to investigate whether specific behavioral treatment (i.e., cognitive-behavior psychotherapy, biofeedback, and so on) can be effective in reducing psychopathological symptoms, improve quality of life, and improving the management of $\mathrm{MOH}$ patients.

\section{DATA AVAILABILITY STATEMENT}

The data that support the findings of this study are available from the corresponding author upon reasonable request.

\section{ETHICS STATEMENT}

This study was designed following the ethical principles of the Declaration of Helsinki and all participants were asked to sign an informed consent. The study was approved by

\section{REFERENCES}

Aaron, R. V., Fisher, E. A., de la Vega, R., Lumley, M. A., and Palermo, T. M. (2019). Alexithymia in individuals with chronic pain and its relation to pain intensity, physical interference, depression and anxiety: a systematic review and meta-analysis. Pain 160, 994-1006. doi: 10.1097/j.pain.0000000000001487

Bayliss, M. S., Dewey, J. E., Dunlap, I., Batenhorst, A. S., Cady, R., Diamond, M. L., et al. (2003). A study of the feasibility of internet administration of a computerized health survey: the headache impact test (HIT). Qual. Life Res. 12, 953-961. doi: 10.1023/a:1026167214355

Beck, A. T., Steer, R. A., Ball, R., and Ranieri, W. (1996). Comparison of beck depression inventories-IA and -II in psychiatric outpatients. J. Pers. Assess. 67, 588-597. doi: 10.1207/s15327752jpa6703_13

Black, A. K., Fulwiler, J. C., and Smitherman, T. A. (2015). The role of fear of pain in headache. Headache 55, 669-679. doi: 10.1111/head.12561

Breslau, N., Lipton, R. B., Stewart, W. F., Schultz, L. R., and Welch, K. M. (2003). Comorbidity of migraine and depression: investigating potential etiology and prognosis. Neurology 60, 1308-1312. doi: 10.1212/01.wnl.0000058907.41080.54

Bressi, C., Taylor, G., Parker, J., Bressi, S., Brambilla, V., Aguglia, E., et al. (1996). Cross validation of the factor structure of the 20-item toronto alexithymia scale: an italian multicenter study. J. Psychosom. Res. 41, 551-559. doi: 10.1016/s00223999(96)00228-0

Buse, D. C., Silberstein, S. D., Manack, A. N., Papapetropoulos, S., and Lipton, R. B. (2013). Psychiatric comorbidities of episodic and chronic migraine. J. Neurol. 260, 1960-1969. doi: 10.1007/s00415-012-6725-x

Ciarrochi, J., Forgas, J. P., and Mayer, J. D. (2001). Emotional Intelligence in Everyday Life: A Scientific Inquiry. Philadelphia, PA: Psychology Press.

Cupini, L. M., De Murtas, M., Costa, C., Mancini, M., Eusebi, P., Sarchielli, P., et al. (2009). Obsessive-compulsive disorder and migraine with medication-overuse headache. Headache 49, 1005-1013. doi: 10.1111/j.1526-4610.2009.01457.x
Campus Bio-Medico University Ethics Committee, approval number 44-18, and registered at AIFA (Italian Drug Agency) with number Eudract 2017-004606-18.

\section{AUTHOR CONTRIBUTIONS}

SiM was involved in the study design, clinical assessment, data collection, analysis, interpretation, and wrote the manuscript. MP was involved in the study design, clinical assessment, data collection, analysis, provided critical review and approval of the manuscript. LQ was involved in the analysis and interpretation of behavioral changes, provided critical review and approval of the manuscript. CA was involved in the study design, data collection, provided critical review and approval of the manuscript. SaM was involved in the interpretation of behavioral changes, provided critical review and approval of the manuscript. GD'A was involved in the study design, data analysis, provided critical review and approval of the manuscript. GC was involved in the study design, data analysis and interpretation, provided critical review and approval of the manuscript. FV was involved in the study design, data collection, data analysis and interpretation, provided critical review and approval of the manuscript. All authors contributed to the article and approved the submitted version.

\section{ACKNOWLEDGMENTS}

We thank Mariafrancesca Zaffaro for her contribution to the data collection.

De Gennaro, L., Cipolli, C., Cherubini, A., Assogna, F., Cacciari, C., Marzano, C. et al. (2011). Amygdala and hippocampus volumetry and diffusivity in relation to dreaming. Hum. Brain Mapp. 32, 1458-1470. doi: 10.1002/hbm.21120

Di Tella, M., and Castelli, L. (2016). Alexithymia in chronic pain disorders. Curr. Rheumatol. Rep. 18:41. doi: 10.1007/s11926-016-0592-x

Eftekhari, A., Zoellner, L. A., and Vigil, S. A. (2009). Patterns of emotion regulation and psychopathology. Anxiety Stress Coping 22, 571-586. doi: 10.1080/10615800802179860

Fossati, A., Di Ceglie, A., Acquarini, E., and Barratt, E. S. (2001). Psychometric properties of an italian version of the barratt impulsiveness scale-11 (BIS-11) in nonclinical subjects. J. Clin. Psychol. 57, 815-828. doi: 10.1002/jclp.1051

Guidetti, V., Galli, F., and Sheftell, F. (2010). Headache attributed to psychiatric disorders. Handb. Clin. Neurol. 97, 657-662. doi: 10.1016/S00729752(10)97055-3

Headache Classification Committee of the International Headache Society (IHS). (2013). The international classification of headache disorders, 3rd edition (beta version). Cephalalgia 33, 629-808. doi: 10.1177/0333102413485658

Koechlin, H., Coakley, R., Schechter, N., Werner, C., and Kossowsky, J. (2018). The role of emotion regulation in chronic pain: a systematic literature review. J. Psychosom. Res. 107, 38-45. doi: 10.1016/j.jpsychores.2018.02.002

Lampl, C., Thomas, H., Tassorelli, C., Katsarava, Z., Laínez, J. M., LantériMinet, M., et al. (2016). Headache, depression and anxiety: associations in the eurolight project. J. Headache Pain 17:59. doi: 10.1186/s10194-0160649-2

Migliore, S., Curcio, G., Porcaro, C., Cottone, C., Simonelli, I., D’Aurizio, G., et al. (2019). Emotional processing in RRMS patients: dissociation between behavioural and neurophysiological response. Mult. Scler. Relat. Disord. 27, 344-349. doi: 10.1016/j.msard.2018.11.019

Migliore, S., Paolucci, M., Quintiliani, L., Altamura, C., D’Aurizio, G., Curcio, G., et al. (2018). Psychopathological profile of medication overuse headache 
patients, drug assumption and degree of disability. Neurol. Sci. 39, 169-170. doi: 10.1007/s10072-018-3390-6

Paolucci, M., Altamura, C., Brunelli, N., Rizzo, A. C., Assenza, F., Pasqualetti, P., et al. (2017). Methylprednisolone plus diazepam i.v. as bridge therapy for medication overuse headache. Neurol. Sci. 38, 2025-2029. doi: 10.1007/s10072017-3098-z

Radat, F., and Lanteri-Minet, M. (2010). What is the role of dependencerelated behavior in medication-overuse headache? Headache 50, 1597-1611. doi: 10.1111/j.1526-4610.2010.01755.x

Radat, F., and Swendsen, J. (2005). Psychiatric comorbidity in migraine: a review. Cephalalgia 25, 165-178. doi: 10.1111/j.1468-2982.2004.00839.x

Sarchielli, P., Corbelli, I., Messina, P., Cupini, L. M., Bernardi, G., Bono, G., et al. (2016). Psychopathological comorbidities in medication-overuse headache: a multicentre clinical study. Eur. J. Neurol. 23, 85-91. doi: 10.1111/ene.12794

Sighinolfi, C., Pala, A. N., Chiri, L. R., Marchetti, I., and Sica, C. (2010). Difficulties in emotion regulation scale (DERS): traduzione e adattamento italiano [difficulties in emotion regulation scale (DERS): the Italian translation and adaptation]. Psicoterapia Cogn. Comport. 16, 141-170.

Spielberger, C. D., Gorsuch, R. L., Lushene, R., Vagg, P. R., and Jacobs, G. A. (1983). Manual for the State-Trait Anxiety Inventory. Palo Alto, CA: Consulting Psychologists Press.
Stewart, W. F., Lipton, R. B., Dowson, A. J., and Sawyer, J. (2001). Development and testing of the migraine disability assessment (MIDAS) questionnaire to assess headache-related disability. Neurology 56, S20-S28. doi: 10.1212/wnl.56. suppl_1.s20

Zarotti, N., Simpson, J., Fletcher, I., Squitieri, F., and Migliore, S. (2018). Exploring emotion regulation and emotion recognition in people with presymptomatic Huntington's disease: the role of emotional awareness. Neuropsychologia 112, 1-9. doi: 10.1016/j.neuropsychologia.2018.02.030

Conflict of Interest: The authors declare that the research was conducted in the absence of any commercial or financial relationships that could be construed as a potential conflict of interest.

Copyright (c) 2020 Migliore, Paolucci, Quintiliani, Altamura, Maffi, D’Aurizio, Curcio and Vernieri. This is an open-access article distributed under the terms of the Creative Commons Attribution License (CC BY). The use, distribution or reproduction in other forums is permitted, provided the original author(s) and the copyright owner(s) are credited and that the original publication in this journal is cited, in accordance with accepted academic practice. No use, distribution or reproduction is permitted which does not comply with these terms. 